\title{
Addenda and Corrigenda: Picard Group of Rings of Coinvariants
}

\section{T. Guédénon}

Published online: 20 January 2009

(C) Springer Science + Business Media B.V. 2009

\section{Erratum to: Algebr Represent Theor (2008) 11: 25-42 DOI 10.1007/s10468-007-9073-2}

We use the same notation and terminology from the paper quoted above. Let $k$ be a field, $A$ a $k$-algebra, $C$ a coalgebra and $H$ a Hopf algebra with bijective antipode $S_{H}$ such that $A$ is a right $H$-comodule algebra with the comodule structure given by $\rho_{A, H}(a)=a_{[0]} \otimes a_{[1]}$ and $C$ is a right $H$-module coalgebra with the right action of $H$ on $C$ denoted by " $\angle$ ". The triple $(H, A, C)$ is called a right-right Doi Hopf datum and $\mathcal{M}(H)_{A}^{C}$ is the category of right-right Doi-Hopf modules. If $C$ is a bialgebra then $A$ is a right $C$-comodule with the comodule structure defined by $\rho_{A, C}(a)=a_{(0)} \otimes a_{(1)}=a_{[0]} \otimes\left(1_{C}<a_{[1]}\right)$. From now on we assume that $C$ is a Hopf algebra with bijective antipode $S_{C}$. In the paper we considered four compatibility conditions (1), (2), (3) and (4). When $C$ is commutative, the conditions (2), (3) and (4) are equivalent. The main results are obtained for $C$ commutative and under the condition (2) or the conditions (1) + (2). Let us recall the conditions (1) and (3):

$$
\begin{gathered}
c^{\prime} S_{C}(c \leftarrow h)=\left(c^{\prime} \leftarrow S_{H}(h)\right) S_{C}(c) ; \forall h \in H, c, c^{\prime} \in C \\
\left(c c^{\prime}\right) \leftarrow h=c\left(c^{\prime} \leftarrow h\right) ; \quad \forall h \in H, c, c^{\prime} \in C
\end{gathered}
$$

Note that for the definition of the condition (3), we can take $C$ to be just a bialgebra. Note also that the condition (3) implies that

$$
c<h=c\left(1_{C}<h\right) ; \quad \forall h \in H, c \in C
$$

The online version of the original article can be found at http://dx.doi.org/10.1007/s10468-007-9073-2.

T. Guédénon $(\varangle)$

110 Penworth Drive S.E., Calgary, AB, T2A 5H4, Canada

e-mail: guedenth@yahoo.ca 
Lemma 1.1 Assume that the condition (3') is satisfied. Then

(1) A is a right $C$-comodule algebra

(2) the category $\mathcal{M}(H)_{A}^{C}$ coincides with the category $\mathcal{M}_{A}^{C}$ of right-right relative ( $A, C)$-Hopf modules.

Proof

(1) See pages 29-30.

(2) Let $M \in \mathcal{M}(H)_{A}^{C}$. For every $m \in M$ and $a \in A$, we have

$$
\begin{aligned}
\rho_{M, C}(m a) & =m_{(0)} a_{[0]} \otimes\left(m_{(1)} \leftarrow a_{[1]}\right) \\
& =m_{(0)} a_{[0]} \otimes m_{(1)}\left(1_{C} \leftarrow a_{[1]}\right) \\
& =m_{(0)} a_{(0)} \otimes m_{(1)} a_{(1)}
\end{aligned}
$$

and so $M \in \mathcal{M}_{A}^{C}$. Conversely, let $M \in \mathcal{M}_{A}^{C}$. For every $m \in M$ and $a \in A$, we have

$$
\begin{aligned}
\rho_{M, C}(m a) & =m_{(0)} a_{(0)} \otimes m_{(1)} a_{(1)} \\
& =m_{(0)} a_{[0]} \otimes m_{(1)}\left(1_{C} \leftarrow a_{[1]}\right) \\
& =m_{(0)} a_{[0]} \otimes\left(m_{(1)} \leftarrow a_{[1]}\right)
\end{aligned}
$$

So $M$ is an object of $\mathcal{M}(H)_{A}^{C}$.

Lemma 1.2 The condition (3') implies the condition (1).

Proof Assume Eq. 3'. For any $h \in H$, we have

$$
\begin{aligned}
1_{C} \epsilon_{H}(h) & =1_{C}\left(\epsilon_{C}\left(1_{C} \leftarrow h\right)\right) \\
& =S_{C}\left(\left(1_{C} \leftarrow h\right)_{1}\right)\left(1_{C} \leftarrow h\right)_{2}=S_{C}\left(1_{C} \leftarrow h_{1}\right)\left(1_{C} \leftarrow h_{2}\right) .
\end{aligned}
$$

Therefore

$$
\begin{aligned}
S_{C}\left(1_{C} \leftarrow h\right) & =S_{C}\left(1_{C} \leftarrow h_{1}\right) \epsilon_{H}\left(h_{2}\right) \\
& =S_{C}\left(1_{C} \leftarrow h_{1}\right) \leftarrow\left(h_{2} S_{H}\left(h_{3}\right)\right) \\
& =\left(S_{C}\left(1_{C} \leftarrow h_{1}\right) \leftarrow h_{2}\right) \leftarrow S_{H}\left(h_{3}\right) \\
& =\left(\left(S_{C}\left(1_{C} \leftarrow h_{1}\right)\left(1_{C} \leftarrow h_{2}\right)\right) \leftarrow S_{H}\left(h_{3}\right)\right. \\
& =\left(\left(S_{C}\left(1_{C} \leftarrow h_{11}\right)\left(1_{C} \leftarrow h_{12}\right)\right) \leftarrow S_{H}\left(h_{2}\right)\right. \\
& =\left(1_{C} \epsilon_{H}\left(h_{1}\right)\right) \leftarrow S_{H}\left(h_{2}\right)=1_{C} \leftarrow S_{H}(h) .
\end{aligned}
$$

Thus for any $h \in H$ and $c, c^{\prime} \in C$, we have

$$
\begin{aligned}
c^{\prime} S_{C}(c \leftarrow h) & =c^{\prime} S_{C}\left(c\left(1_{C} \leftarrow h\right)\right) \\
& =c^{\prime} S_{C}\left(1_{C} \leftarrow h\right) S_{C}(c)=c^{\prime}\left(1_{C} \leftarrow S_{H}(h)\right) S_{C}(c) \\
& =\left(c^{\prime} \leftarrow S_{H}(h)\right) S_{C}(c)
\end{aligned}
$$

which is the condition (1).

空 Springer 
Note that in Lemma 1.1, we can assume that $C$ is just a bialgebra.

What is behind this note is the following: while many results of the paper are established for the category of Doi-Hopf modules, the main results concern the category of relative Hopf modules.

Finally the following misprints have occurred in the paper:

Page 28: The comodule structure defined on $A$ requires $C$ to be a bialgebra.

Page 30: "Definition 2.2" should be "Definition 2.2 (see [9])"; that is " Definition 2.2 (see [9])" which appears in the statements of Definition 2.2 is not correct.

Page 33: In Lemma 3.2, " $\mathcal{M}_{A}$ " should be “ $\mathcal{M}_{B}$ ". 